\title{
Consumo de alcohol y factores de riesgo de conductas autolesivas en adolescentes españoles
}

\author{
Alcohol use and risk factors for self-harm \\ behavior in Spanish adolescents
}

\author{
Matilde Bousoño*, Susana Al-Halabí**********, Patricia Burón******, Marlen Garrido*, Eva M ${ }^{\text {a }}$ \\ Díaz-Mesa*,****, Gonzalo Galván*****, Leticia García-Állvarez**************, Ángela Velasco*****, \\ Julia Rodríguez-Revuelta*,****,******,*******, Camilla Wasserman********,*********, \\ Vladimir CARli********, Christina Hoven*********, Marco SARChiapone*********, DanUta \\ WAsserman*******, Manuel Bousoño*,***,****, Ma Paz García-Portilla*,***,****,******,*******, \\ Celso Iglesias $* * * *, * * * *, * * * * * *, * * * * * * *$, Pilar Alejandra SÁIz*,***,****,******,*******, Julio \\ BobES $* * * *, * * * *, * * * * * *, * * * * * * *$.
}

* Área de Psiquiatría. Universidad de Oviedo. ** Departamento de Psicología. Universidad de Oviedo. *** Centro de Investigación Biomédica en Red de Salud Mental (CIBERSAM). **** Instituto de Neurociencias del Principado de Asturias (INEUROPA). ***** Universidad Cooperativa de Colombia. Colombia. ****** Instituto de Investigación Sanitaria del Principado de Asturias (ISPA). ******* Servicio de Salud del Principado de Asturias (SESPA). ******** National Centre for Suicide Research and Prevention of Mental Ill-Health, Karolinska Institutet, Stockholm. Sweden. ********* Division of Child and Adolescent Psychiatry and Department of Psychiatry, New York State Psychiatric Institute. Columbia University, New York. USA. Department of Epidemiology, Mailman School of Public Health. Columbia University, New York. USA. ********** Department of Medicine and Health Science. University of Molise, Campobasso. Italy. National Institute for Health, Migration and Poverty, Rome. Italy.

\section{Resumen}

Las conductas autolesivas en niños y adolescentes constituyen un importante problema de salud pública con cifras de prevalencia en la población clínica entre el 40 y $80 \%$. Los objetivos del estudio son analizar y comparar las submuestras españolas de dos trabajos, SEYLE y WE-STAY, para conocer la prevalencia, los patrones de autolesión y los factores asociados a las conductas autolesivas, en particular el consumo de alcohol o drogas. Los cuestionarios utilizados en ambos estudios fueron la Encuesta Global de Salud Escolar (GSHS), el Inventario de Depresión de Beck (BDI-II), el Cuestionario de Fortalezas y Dificultades (SDQ). Los comportamientos autolesivos fueron evaluados con una versión modificada de 6 ítems basada en el Inventario de Autolesiones Deliberadas (DSHI). La independencia de las variables categóricas del estudio se evaluó mediante la prueba Ji-Cuadrado. El cambio en el riesgo relativo de autolesión entre el estudio SEYLE y WE-STAY, se evaluó a través del cálculo de odds ratio (OR). Se calcularon dos modelos de regresión logística diferentes con el fin de establecer los factores asociados con comportamientos autolesivos en cada estudio. En el presente estudio las tasas de DSH varían en función del estudio y del sexo en un rango entre $0,58 \%$ y $2,08 \%$, presentando patrones de autolesiones diferentes según el sexo, los hombres se autolesionaron más frecuentemente mediante golpes autoinfligidos y quemaduras, mientras que las mujeres se hicieron más frecuentemente cortes. La presencia de síntomas depresivos y el consumo de alcohol fueron los factores asociados de forma más robusta a un mayor riesgo de DSH.

Palabras clave: Conductas autolesivas; Adolescentes; Depresión; Consumo de alcohol; SEYLE; WE-STAY.

\begin{abstract}
Self-harm behaviors in children and adolescents constitute an important public health problem with prevalence figures in the clinical population between 40 and $80 \%$. The objectives of the study were to analyze and compare the Spanish sub-samples of two studies, SEYLE and WE-STAY to determine prevalence, self-harm patterns and factors associated with self-harm behaviors, notably the use of alcohol or drugs. The questionnaires used in both studies were the Global School Health Survey (GSHS), the Beck Depression Inventory (BDI-II), the Strengths and Difficulties Questionnaire (SDQ). The self-harm behaviors were evaluated with a modified 6-item version of s the Deliberate Self-Harm Inventory (DSHI). The independence of the study's categorical variables was assessed using the Chi-square test. The change in the relative risk of self-harm between the SEYLE study and WE-STAY was evaluated through the odds ratio (OR) calculation. Two different logistic regression models were calculated in order to establish the factors associated with self-harm behaviors in each study. In the present study, the rates of DSH vary according to study and sex, ranging from $0.58 \%$ to $2.08 \%$, and different patterns of selfharm are evidenced by sex, with males self-injuring more frequently by self-inflicted blows and burns, while young women more often cut themselves. The presence of depressive symptoms and alcohol use were the factors most strongly associated with an increased risk of DSH.
\end{abstract}

Key Words: Self-harm behaviors; Adolescents; Depression; Alcohol consumption; SEYLE; WE-STAY.

Recibido: Septiembre 2018; Aceptado: Noviembre 2018.

Enviar correspondencia a: Julio Bobes García.

Área de Psiquiatría. Facultad de Medicina. Julián Clavería 6- 3º , 33006, Oviedo.

E-mail: bobes@uniovi.es. 


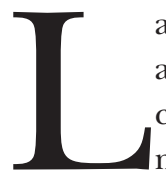

a adolescencia es un periodo de la vida en el que aparecen conductas de riesgo que se han relacionado tanto con características del proceso de maduración cerebral como con factores sociales y culturales propios de esta etapa (McCormick, Qu y Telzer, 2017; Somerville, Hare y Casey, 2011). Entre los riesgos asumidos con mayor frecuencia por los adolescentes están las conductas autolesivas (Kaess, Fischer-Waldschmidt, Resch y Koenig, 2017).

La autolesión es el acto de dañarse a uno mismo deliberadamente causándose una herida física, poniéndose en situaciones de riesgo o siendo negligente en el autocuidado (Bifulco et al., 2014). La "autolesión no suicida” (non suicidal self-injury -NSSI-) ha sido propuesta como una de las "categorías diagnósticas del DSM-5 que requieren mayor estudio", y se define como el daño intencional autoinfligido a la superficie del cuerpo sin intención suicida consciente y con propósito no aceptado socialmente; incluye conductas tales como cortarse, quemarse, morderse o erosionar la piel (American Psychiatric Association, 2013; Zetterqvist, 2015). La "autolesión deliberada" (deliberate self-harm -DSH-), es un concepto parecido en el que no se valora la existencia o no de intencionalidad suicida ( $\mathrm{Pa}-$ ttison y Kahan, 1983), y que puede ser directa (cortarse o golpearse) e indirecta o conducta autodestructiva (beber alcohol, fumar tabaco, etc.) (Nock, 2010).

Las conductas autolesivas en niños y adolescentes constituyen un importante problema de salud pública con tasas de incidencia anual en niños y adolescentes entre 12,3 y 37,4 por 10000 (Morgan et al., 2017). El establecimiento de cifras de prevalencia de autolesiones en la población general es difícil debido a la escasez de datos, pero la evaluación de los estudios disponibles realizados en distintos países permite concluir que se trata de un fenómeno frecuente en adolescentes y adultos jóvenes en la población general (entre el 13 y el 42\%) y más frecuente aún en poblaciones clínicas (entre el 40\% y el 80\%) (Lenkiewicz, Racicka y Bryńska, 2017). La prevalencia no varía en función de factores culturales, estatus socioeconómico o área de residencia (Lenkiewicz, Racicka y Bryńska, 2017; Muehlenkamp, Claes, Havertape y Plener, 2012).

El efecto del sexo en las conductas autolesivas es controvertido, con trabajos que muestran una mayor frecuencia en mujeres (ratio mujer:hombre que puede llegar a 6,5:1); y otros que no encuentran diferencias entre sexos (Bresin y Schoenleber, 2015; Sornberger, Heath, Toste y McLouth, 2012; Whitlock et al., 2011). Algún estudio ha relacionado el género y las autolesiones teniendo en cuenta otros factores asociados como: el consumo de alcohol de los padres, que se asocia con más riesgo de autolesiones en los hombres; o el grado de urbanicidad que muestra una mayor prevalencia de autolesiones no suicidas en mujeres en zona rural que no se mantiene en las zonas urbanas (Pisinger, Hawton y Tolstrup, 2018; Yang y Feldman, 2017).
La edad sí parece que tiene una asociación clara con las conductas autolesivas, que suelen empezar a manifestarse entre los 12 y los 14 años y cuya frecuencia disminuye al aumentar la edad (Cipriano, Cella y Cotrufo, 2017; Hawton y Harriss, 2008; Lenkiewicz, Racicka y Bryńska 2017). También se ha encontrado asociación entre autolesiones y psicopatología. La presencia de psicopatología externalizante en los adolescentes (relacionadas con las manifestaciones de agresividad, falta de atención, desobediencia y conducta delictiva) se asocia con más autolesiones no suicidas, y los adolescentes que se ven sometidos a situaciones vitales generadoras de frustración o vacío existencial presentan más conductas suicidas (Blasco-Fontecilla, 2018; Meszaros, Horvath y Balazs, 2017).

Recientemente, dos estudios multicéntricos, SEYLE (Wasserman et al., 2010) y WE-STAY (Strittmatter et al., 2015) han investigado en profundidad las conductas autolesivas y suicidas en adolescentes de varios países europeos entre los que se encuentra España. En el presente trabajo se analizan las submuestras españolas de los citados estudios para conocer la prevalencia, el tipo de autolesión y los factores de riesgo asociados a las conductas autolesivas. Además, se han comparado los datos de ambos estudios entre sí, partiendo de la hipótesis de que, por su cercanía en el tiempo de realización y por sus similitudes metodológicas, deberían arrojar resultados similares.

\section{Método}

En el presente trabajo se analizan los datos de las submuestras españolas de dos estudios realizados en el ámbito europeo: el estudio Saving and Empowering Young Lives in Europe (SEYLE) realizado en los años 2009 y 2010, y el estudio Working in Europe to Stop Truancy Among Youth (WESTAY) realizado en 2011 y 2012. Los procedimientos utilizados en los estudios SEYLE y WE-STAY han sido objeto de publicaciones específicas (Carli et al., 2014; Strittmatter et al., 2015; Wasserman et al., 2010).

\section{Participantes}

La muestra (submuestra española) del estudio SEYLE está compuesta por 1026 adolescentes con una edad media (EM) de 14,52 años y una desviación estándar (DE) de 0,70 años; el $51,66 \%$ de la muestra eran hombres. Los participantes fueron reclutados en 12 centros educativos públicos seleccionados de forma aleatoria entre los existentes en la Comunidad Autónoma del Principado de Asturias, teniendo en cuenta los criterios de inclusión y exclusión del proyecto SEYLE (Bousoño et al., 2017; Wasserman et al., 2010). La muestra (submuestra española) del estudio WESTAY está compuesta por 1409 adolescentes, con una EM de 15,16 años ( $\mathrm{DE}=1,23$ años), de los que el 48,83\% eran hombres; fueron reclutados en 26 centros educativos públicos del Principado de Asturias. Los dos estudios se realizaron en centros educativos diferentes. 


\section{Procedimiento}

En cumplimiento de las normas que rigen la investigación en población joven, previamente al inicio de ambos estudios se obtuvo la autorización del fiscal de menores y la aprobación del Comité Ético de Investigación Clínica del Principado de Asturias. En los dos casos, las autoridades escolares locales otorgaron permiso para acceder a los centros educativos seleccionados y los sujetos de estudio otorgaron el asentimiento y consentimiento informado, según fuera requerido. Se aplicó un cuestionario estructurado de autoinforme a los sujetos participantes y los datos recogidos fueron anonimizados. Las evaluaciones fueron realizadas en horario escolar y recogen una amplia gama de factores demográficos, psicológicos y sociales.

\section{Métodos de evaluación}

Para la valoración del consumo de sustancias se utilizó el cuestionario Global School-based Student Health Survey -GSHS- (World Health Organization, 2015), estableciendo los siguientes puntos de corte: para considerar "consumo de alcohol" se consideró como criterio el consumir cualquier cantidad de alcohol dos o más veces a la semana; para el "consumo de drogas", haber usado drogas ilegales al menos tres veces a lo largo de la vida y para el "consumo de tabaco" fumar más de diez cigarrillos al día (Bousoño et al., 2019).

Para evaluar los "síntomas depresivos" se utilizó el inventario de Beck (BDI-II) (Beck, Steer, Ball y Ranieri, 1996), considerando que una puntuación igual o mayor de 20 suponía "riesgo de depresión". Para el presente estudio se ha utilizado una versión modificada, el BDI-II, eliminando el ítem "pérdida de la libido" ya que se consideró una pregunta inapropiada para la población objeto de estudio. La omisión de esta pregunta no afecta a la fiabilidad o validez al instrumento (Byrne, Stewart y Lee, 2004).

Para evaluar la psicopatología se empleó el Cuestionario de Capacidades y Dificultades (SDQ) (Goodman, Meltzer y Bailey, 2003) que valora síntomas emocionales, problemas de conducta, hiperactividad/falta de atención, problemas de relación entre pares y comportamiento prosocial. Los puntos de corte establecidos fueron: una puntuación mayor o igual que 7 para los síntomas emocionales, una puntuación mayor o igual que 5 para los "problemas de conducta" y una puntuación mayor o igual que 7 para la "hiperactividad". En el caso de los "problemas con los compañeros", el punto de corte se fijó en una puntuación mayor o igual que 6, mientras que la "falta de comportamiento prosocial" se definió con una puntuación menor o igual que 4 (Carli et al., 2014).

Las DSH fueron evaluadas con una versión modificada de 6 ítems (Brunner et al., 2014) del Inventario de autolesiones deliberadas (DSHI) (Gratz, 2001) que valora diferentes formas de comportamiento autolesivo. Se utilizó como punto de corte el que los estudiantes informaran de estas conductas 3 veces o más en el último año (Carli et al., 2014). Las 6 preguntas (P1, P2...) contenidas en el cuestionario son: P1: durante el último año ¿alguna vez te has cortado intencionalmente las muñecas, los brazos $\mathrm{u}$ otras partes de tu cuerpo, o clavado objetos afilados en la piel, como agujas, alfileres, pins, grapas (No incluyas tatuajes, agujeros en las orejas, pinchazos para drogarte o piercings)?; P2: durante el último año ¿alguna vez te has quemado intencionalmente con un cigarrillo, un mechero o una cerilla?; P3: durante el último año ¿alguna vez te has cortado intencionalmente la piel haciendo palabras, dibujos, diseños u otras marcas, o te has arañado tanto que sangraste o te hiciste cicatrices?; P4: durante el último año ¿alguna vez has impedido intencionalmente una cicatrización o te has mordido tan fuerte que te has rasgado la piel?; P5: durante el último año ¿alguna vez te has golpeado la cabeza u otra parte del cuerpo causándote una contusión?; P6 durante el último año ¿alguna vez te has herido intencionalmente de alguna forma de las anteriormente mencionadas, de tal manera que has tenido que ser hospitalizado o ha sido lo suficientemente grave como para recibir tratamiento médico?.

\section{Análisis estadístico}

Con el fin de comprobar la independencia de las variables categóricas del estudio, se aplicó el test de Ji-Cuadrado. Además, también se calcularon dos modelos de regresión logística, uno para el estudio SEYLE y otro para WE-STAY, en el que la variable dependiente fue el riesgo de autolesión y las variables independientes fueron la edad, el sexo, SDQ síntomas emocionales, SDQ problemas de conducta, SDQ hiperactividad, SDQ problemas con los compañeros, SDQ falta de comportamiento prosocial, BDI-II, consumo de tabaco, consumo de alcohol y consumo drogas.

\section{Resultados}

Cumplían criterios de conductas auto-lesivas deliberadas (Total DSHI $\geq 3)$, el 1,56\% (n=16) de participantes del estudio SEYLE, frente al 0,92\% $(\mathrm{n}=13)$ del WE-STAY $\left(X^{2}=\right.$ $2,046, \mathrm{gl}=1, \mathrm{p}<0.153)$.

Analizando las DSH en función del sexo, en las mujeres no se encontraron diferencias de prevalencia entre ambos estudios $[1,01 \%(n=5)$ vs $1,26 \%(n=9)$, respectivamente; $\left.X^{2}=0,149, \mathrm{gl}=1, \mathrm{p}=0,699\right]$, mientras que la prevalencia en hombres si difiere de modo estadísticamente significativo $\left[2,08 \%(n=11)\right.$ vs $0,58 \%(n=4)$, respectivamente; $X^{2}=$ $5,494, \mathrm{gl}=1, \mathrm{p}=0,019]$. Cabe reseñar que cuando ambos estudios son evaluados por separado no se encuentran diferencias en la prevalencia de conductas auto-lesivas entre hombres y mujeres en ninguno de los dos estudios (SEYLE: 2,08 vs $1,01 \%, X^{2}=1,902, \mathrm{gl}=1, \mathrm{p}=0,168$; WE-STAY: $0,58 \%$ vs $1,26 \%, X^{2}=1,713$, gl= $\left.1, \mathrm{p}<0,191\right)$.

En la Tabla 1 se muestran los resultados del cuestionario DSHI, ítem a ítem, en cada estudio en función del sexo. Como puede observarse en dicha tabla, en el estudio 
SEYLE, la frecuencia de hombres que superan el punto de corte (3 veces o más en el último año) en los ítems 2 (quemaduras autoinfligidas) y 5 (golpes / contusiones) es significativamente superior a la de las mujeres. Mientras que en el estudio WE-STAY, las mujeres superan el punto de corte (3 veces o más en el último año) en los ítems 1 (cortes o uso de objetos afilados incisivos) y 3 (cortes simulando palabras, dibujos, etc.) con una frecuencia significativamente superior a la de los hombres.

Tabla 1. Comparación del tipo de autolesiones entre los estudios SEYLE y WE-STAY, según la puntuación individual en los diferentes ítems del cuestionario DSHI, desagregado por sexo.

\begin{tabular}{|c|c|c|c|c|c|c|}
\hline & & $\begin{array}{c}\text { Total n } \\
\text { (\%) }\end{array}$ & $\begin{array}{c}\text { Hombres n } \\
\text { (\%) }\end{array}$ & $\begin{array}{l}\text { Mujeres n } \\
\text { (\%) }\end{array}$ & $X^{2}(\mathrm{gl})$ & p \\
\hline \multirow[t]{2}{*}{ P1 } & SEYLE & $32(3,12 \%)$ & $14(2,64 \%)$ & $18(3,63 \%)$ & 0,827 (1) & 0,363 \\
\hline & WE-STAY & $25(1,77 \%)$ & $6(0,8$ & 16 & (1) & 0,041 \\
\hline \multirow[t]{2}{*}{ P2 } & SEYLE & $27(2,63 \%)$ & $19(3,58 \%)$ & $8(1,61 \%)$ & 3,889 (1) & 0,049 \\
\hline & WE-STAY & 28 (1,99\%) & 11 (1,59\%) & 7 (0,98\%) & $1,101(1)$ & 0,294 \\
\hline \multirow[t]{2}{*}{ P3 } & SEYLE & $32(3,12 \%)$ & $17(3,21 \%)$ & $15(3,02 \%)$ & 0,029 (1) & 0,866 \\
\hline & WE-STAY & $35(2,48 \%)$ & $8(1,15 \%)$ & $27(3,77 \%)$ & 9,689 (1) & 0,002 \\
\hline \multirow[t]{2}{*}{ P4 } & SEYLE & 26( & 15( & 11 & 0,389 (1) & 0,533 \\
\hline & WE-STAY & 27 (1,92\%) & $11(1,59 \%)$ & $16(2,23 \%)$ & 0,721 & 0,396 \\
\hline \multirow[t]{2}{*}{ P5 } & SEYLE & $21(2,05 \%)$ & $17(3,21 \%)$ & $4(0,81 \%)$ & 7.368 (1) & 0,007 \\
\hline & WE-STAY & $17(1,21 \%)$ & $8(1,15 \%)$ & $9(1,26 \%)$ & $0,022(1)$ & 0,883 \\
\hline \multirow[t]{2}{*}{ P6 } & SEYLE & $5(0,49 \%)$ & $4(0,75 \%)$ & $1(0,20 \%)$ & 1,6 & 0,204 \\
\hline & WE-STAY & $3(0,21 \%)$ & $1(0,14 \%)$ & $2(0,28 \%)$ & 0,289 (1) & 0,591 \\
\hline \multirow[t]{2}{*}{ DSHI $\geq 3$} & SEYLE & $16(1,56 \%)$ & $11(2,08 \%)$ & $5(1,01 \%)$ & 1,902 (1) & 68 \\
\hline & WE-STAY & $13(0,92 \%)$ & $4(0,58 \%)$ & $9(1,26 \%)$ & $1,713(1)$ & 0,191 \\
\hline
\end{tabular}

Nota. DSHI = Inventario de autolesiones deliberadas.

Comparando los resultados de ambos estudios entre sí, se observa un porcentaje significativamente superior de respuestas que superan el punto de corte (3 veces o más en el último año) en los dos primeros ítems del cuestionario (cortes o uso de objetos afilados incisivos y quemaduras autoinfligidas) en el estudio SEYLE frente al WE-STAY (P1: 3,12 vs 1,56\%, $X^{2}=6,642, \mathrm{gl}=1, \mathrm{p}=0,010 ; \mathrm{P} 2: 2,63 \%$ vs $1,28 \%, X^{2}=6,001, \mathrm{gl}=1, \mathrm{p}<0,014$ ) (Figura 1).

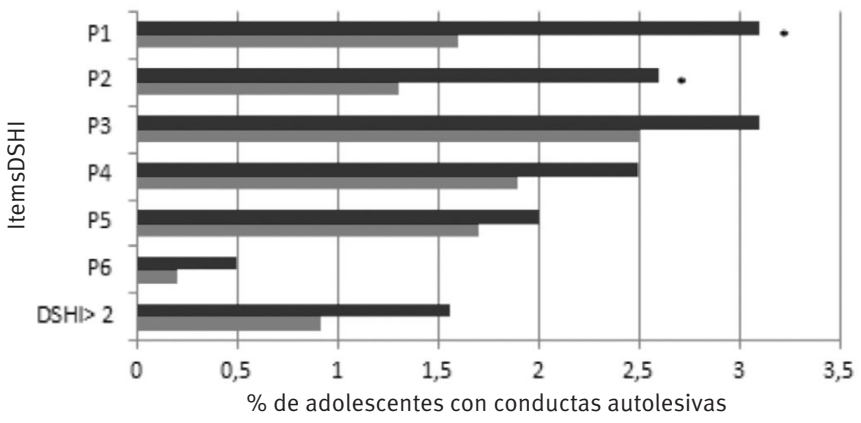

Nota. ${ }^{*} \mathrm{p} \leq$ 0,050; DSHI = Inventario de autolesiones deliberadas.

Figura 1. Comparación del tipo de autolesiones entre los estudios SEYLE y WE-STAY, según la puntuación individual en los diferentes ítems del cuestionario DSHI.
En la Figura 2 (A y B), se muestra la distribución de respuestas positivas, ítem a ítem en cada estudio en función del sexo. Como puede en la Figura 2A, los hombres participantes en el estudio SEYLE, presentan para todos los ítems (excepto el 4 y el 6) un porcentaje significativamente más elevado de respuestas positivas (P1: 2,64 vs 0,87\%, $X^{2}=$ 5,803, $\mathrm{gl}=1, \mathrm{p}=0,016$; $\mathrm{P} 2: 3,58 \%$ vs $1,59 \%, X^{2}=4,915, \mathrm{gl}=$ $1, \mathrm{p}=0,027 ; \mathrm{P} 3: 3,21 \%$ vs $1,15 \%, X^{2}=6,226, \mathrm{gl}=1, \mathrm{p}=0,013$; P5: $3,21 \%$ vs $\left.1,15 \%, X^{2}=6,226, \mathrm{gl}=1, \mathrm{p}=0,013\right) \mathrm{y}$, de igual modo existe un mayor porcentaje de hombres con puntuación DSHI $\geq 3$ en el SEYLE que en el WE-STAY $(2,08$ vs $\left.0,58 \%, X^{2}=5,494, \mathrm{gl}=1, \mathrm{p}=0,019\right)$. En cambio, en las mujeres (Figura 2B), no se observan diferencias en el porcentaje de respuestas positivas en ninguno de los ítems, ni en el porcentaje de mujeres con puntuación DSHI $\geq 3$, que son similares en ambos estudios.

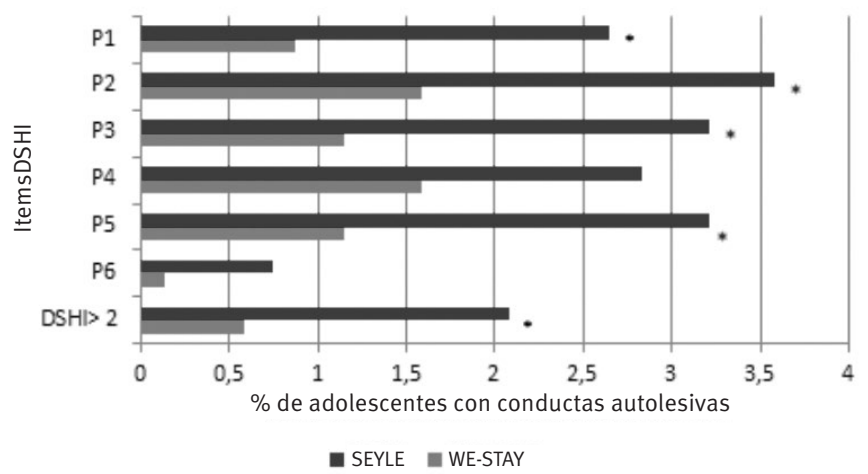

Figura $2 A$. Hombres.

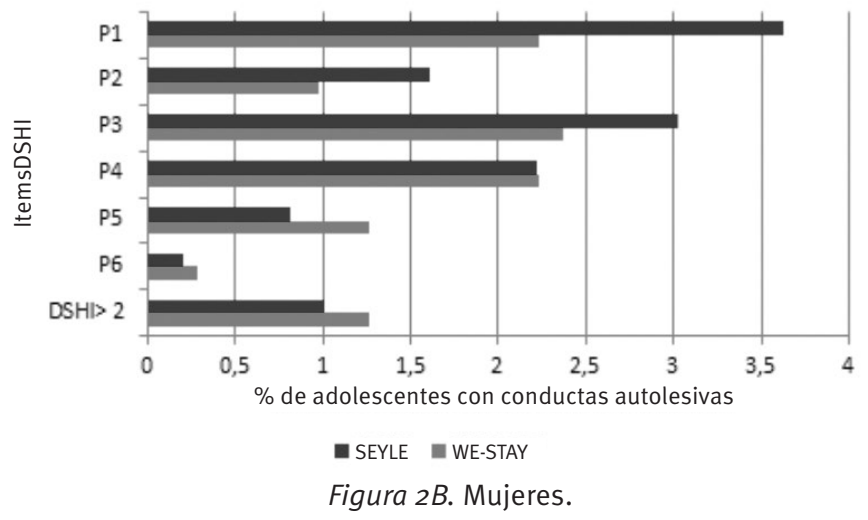

Nota. ${ }^{*} \mathrm{p} \leq 0,050 ; \mathrm{DSHI}=$ Inventario de autolesiones deliberadas.

Figura 2. Tipo de autolesiones observadas en los estudios SEYLE y WE-STAY, según la puntuación individual en los diferentes ítems del cuestionario DSHI, desagregados por sexo

Con el fin de determinar los factores asociados a la realización de conductas auto-lesivas se realizan dos análisis de regresión logística independientes (uno para cada estudio), con el fin de tratar de replicar (o no) los resultados en dos muestras independientes. En ambos casos se utilizan como variables de control la edad y el sexo.

Como puede verse en la Tabla 2, los factores asociados con comportamientos auto-lesivos en el estudio SEYLE 
(tras controlar por edad y sexo), fueron la presencia de hiperactividad/falta de atención (puntuación SDQ hiperactividad / falta de atención $\geq 7)(\mathrm{OR}=2,367$, IC 95\%= 1,389-4,033; $\mathrm{p}=$,002), problemas de relación con pares (puntuación SDQ relación con pares $\geq 6)(\mathrm{OR}=3,096$, IC 95\%= 1,230-7,797; $\mathrm{p}=, 024$ ), presencia de depresión (puntuación $\mathrm{BDI}-\mathrm{II} \geq 20) \quad(\mathrm{OR}=4,046, \quad \mathrm{IC} 95 \%=2,321-7,050$; $\mathrm{p}<, 001)$ y consumo de alcohol ( $\geq 2$ veces/semana) (OR= $2,362$, IC $95 \%=1,240-4,499 ; \mathrm{p}=, 012)$, drogas ilegales $(\geq$ 3 consumos/vida) $(\mathrm{OR}=2,843$, IC $95 \%=1,360-5,944 ; \mathrm{p}=$
,007), y tabaco (> 10 cigarros/día) $(\mathrm{OR}=3,464$, IC 95\%= $1,632-7,353 ; \mathrm{p}=, 002)$.

Sin embargo, en la Tabla 3, puede verse cómo en el WESTAY, sólo existen tres factores asociados a los comportamientos auto-lesivos, que fueron la falta de comportamiento pro-social (SDQ pro-social $\leq 4) \quad(\mathrm{OR}=2,809$, IC $95 \%=$ 1,199-6,581; $\mathrm{p}=, 027)$, la presencia de depresión (puntuación $\mathrm{BDI}-\mathrm{II} \geq 20) \quad(\mathrm{OR}=6,357, \mathrm{IC} 95 \%=3,613-11,183$; $\mathrm{p}<, 001)$ y consumo de alcohol ( $\geq 2$ veces/semana) $(\mathrm{OR}=$ 2,353, IC 95\%=1,259-4,399; $\mathrm{p}=, 010)$.

Tabla 2. Factores asociados con comportamientos autolesivos en la muestra española del estudio SEYLE.

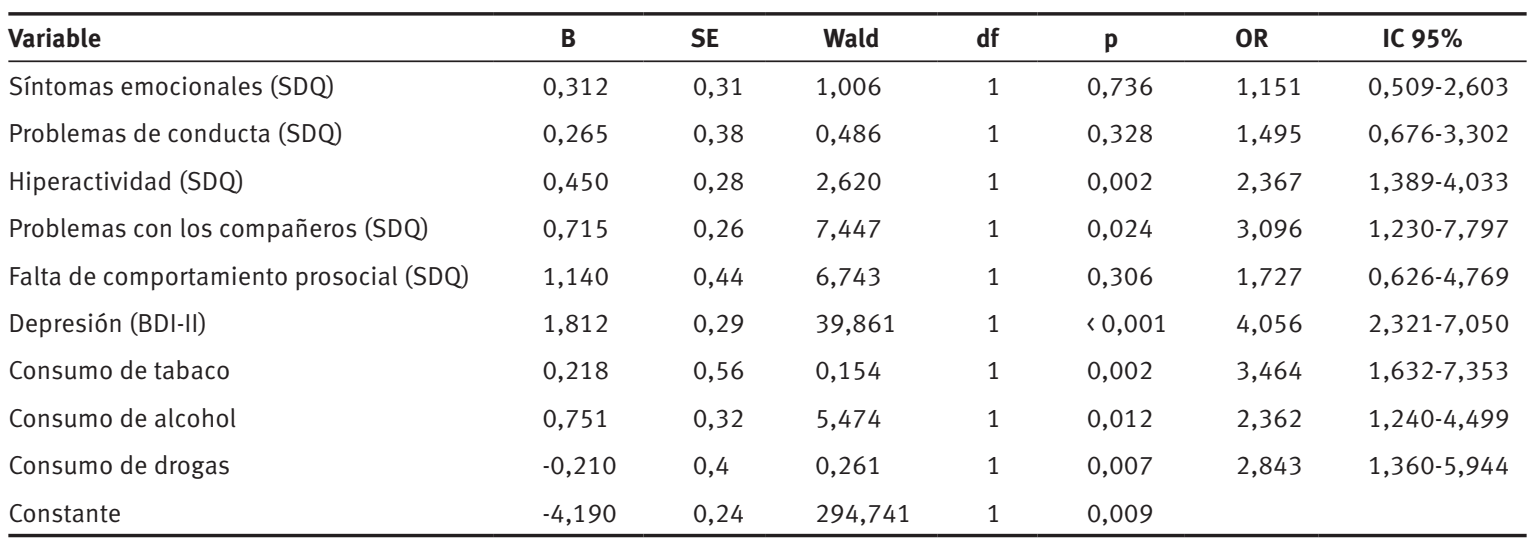

Nota. $\mathrm{SDQ}=$ Cuestionario de Capacidades y Dificultades; $\mathrm{BDI}-\mathrm{II}=$ Inventario de depresión de Beck; $\mathrm{SE}=$ Error estándar; $\mathrm{df}=$ grados de libertad; $\mathrm{OR}=$ Odds ratio; $\mathrm{IC}=$ Intervalo de confianza.

Tabla 3. Factores asociados con comportamientos autolesivos en la muestra española del estudio WE-STAY.

\begin{tabular}{|c|c|c|c|c|c|c|c|}
\hline Variable & B & SE & Wald & df & $\mathbf{p}$ & OR & IC $95 \%$ \\
\hline Problemas de conducta (SDQ) & 0,401 & 0,41 & 0,980 & 1 & 0,454 & 1,330 & $0,639-2,764$ \\
\hline Hiperactividad (SDQ) & 0,862 & 0,27 & 10,043 & 1 & 0,088 & 1,614 & $0,929-2,771$ \\
\hline Problemas con los compañeros (SDQ) & 1,130 & 0,47 & 5,756 & 1 & 0,180 & 1,776 & $0,791-3,987$ \\
\hline Consumo de tabaco & 1,242 & 0,38 & 10,461 & 1 & 0,593 & 1,354 & $0,457-4,017$ \\
\hline Consumo de alcohol & 0,859 & 0,33 & 6,817 & 1 & 0,010 & 2,353 & $1,259-4,399$ \\
\hline Consumo de drogas & 1,045 & 0,38 & 7,724 & 1 & 0,637 & 0,828 & $0,375-1,829$ \\
\hline
\end{tabular}

Nota. $\mathrm{SDQ}=$ Cuestionario de Capacidades y Dificultades; $\mathrm{BDI}-\mathrm{II}=$ Inventario de depresión de Beck; $\mathrm{SE}=$ Error estándar; $\mathrm{df}=$ grados de libertad; $\mathrm{OR}=$ Odds ratio; $\mathrm{IC}=$ Intervalo de confianza.

\section{Discusión}

En el presente trabajo se han estudiado las DSH, sus características y los factores de riesgo asociados a las mismas en el grupo de adolescentes españoles incluidos en dos estudios multicéntricos europeos (SEYLE y WE-STAY).

Se encontraron tasas de prevalencia de DSH de 1,56\% y $0,92 \%$, que se sitúan en el límite inferior de las tasas presentadas por las muestras del resto de países europeos participantes en los estudios SEYLE y WE-STAY, entre los cuales Rumanía presentó la tasa más baja $(1,9 \%)$ y Alemania la tasa más elevada $(10,4 \%)$.
Las tasas de prevalencia encontradas variaron en función de los grupos de población analizados, con diferencias que alcanzaron la significación estadística cuando se compararon los hombres de ambos estudios (los hombres del estudio SEYLE presentaron una prevalencia de DSH 3,5 veces superior a los del WE-STAY). Estudios previos también han encontrado cifras de prevalencia de conductas autolesivas dispares, que van desde el 3\% (Taliaferro y Muehlenkamp, 2015) hasta el 11,5\% (Madge et al., 2008); justificándose las diferencias, fundamentalmente, por la heterogeneidad del concepto de conducta autolesiva uti- 
lizado y por diferencias en los métodos utilizados para la detección de casos (Hargus, Hawton y Rodham, 2009; Silverman, Berman, Sanddal, O’Carroll y Joiner, 2007). Los estudios comparados en el presente trabajo también presentaron variaciones en las tasas a pesar de haber sido realizados en momentos temporales próximos, con muestras de características similares y con métodos de evaluación similares, por lo que es probable que, además de las diferencias conceptuales y metodológicas, haya otras circunstancias que influyan en la variabilidad de la prevalencia de conductas autolesivas, como pudiera ser la escasa frecuencia del fenómeno estudiado que hace que hace que pequeñas variaciones en el número absoluto de afectados se traduzca en cambios importantes en las tasas.

Las tasas de autolesiones observadas en las muestras españolas de los estudios SEYLE y WE-STAY son inferiores a las observadas en otras muestras españolas. Un estudio en el que participaron 1.171 estudiantes de ESO (518 chicos y 653 chicas) de entre 12 y 16 años de escuelas públicas y privadas de Barcelona y alrededores (Kirchner, Ferrer, Forns y Zanini, 2011) encontró un 11,4\% de prevalencia en el año previo de DSH. En este trabajo se utilizó un método de evaluación diferente, la escala Youth Self Report (YSR) (Achenbach, 1991) y de las contestaciones positivas al ítem que investigaba las conductas autolesivas el 2,9\% contestaron "completamente cierto" y el 8,5\% "posiblemente cierto". Otro estudio reciente realizado sobre una muestra amplia de 1664 adolescentes (edad media= 16,12 años) de la Comunidad Autónoma de La Rioja encontró que un 4,1\% había intentado quitarse la vida en el año anterior (Fonseca-Pedrero et al., 2018), aunque no entran a valorar las conductas autolesivas. Otro trabajo español aporta cifras del 21,7\% de autolesiones deliberadas a lo largo de la vida, aunque los datos no son comparables por tratarse de una muestra clínica (adolescentes atendidos en las consultas ambulatorias de psiquiatría de un hospital general) (Díaz de Neira et al., 2015).

El análisis de las tasas de prevalencia desagregadas por sexo de los estudios SEYLE y WE-STAY, como sucede con los datos de estudios previos, no aporta resultados claros que permitan establecer de forma concluyente la influencia del sexo en las DSH. Sin embargo, sí se encontraron patrones de autolesión diferentes entre sexos: en uno de los estudios los hombres se autolesionaron más frecuentemente mediante golpes autoinfligidos y quemaduras, mientas que las mujeres se hicieron más asiduamente cortes o escoriaciones. Parece que el patrón masculino (golpes, quemaduras) tiene características de impulso inmediato liberador de tensión, mientras que el femenino es una conducta más compleja, menos impulsiva, más compulsiva y en la que, además del dolor, se busca la realización de marcas que denoten una identidad o personalidad propia diferenciada, a la manera de los tatuajes o los piercings, es decir, que tengan un significado identitario.
Los factores de riesgo asociados a la presencia de DSH difirieron entre los dos estudios analizados, lo que resulta coherente con el hecho de que los factores de riesgo de conductas autolesivas demuestran una especificidad muy baja y un escaso valor predictivo (Barrigón y Baca-García, 2018). Aparecieron como factores de riesgo asociados significativamente a la DSH en solo uno de los estudios: la hiperactividad, los problemas con los compañeros, los consumos de tabaco, los consumos de drogas y la falta de comportamiento prosocial. Factores de riesgo asociados a las DSH en los dos estudios fueron: la presencia de síntomas depresivos y el consumo de alcohol.

La presencia de síntomas depresivos y el consumo de alcohol aparecen como los factores que más se asocian a las DSH. El ánimo depresivo se ha mostrado como un factor asociado a las conductas autolesivas en la mayoría de estudios realizados en población adolescente (Dougherty et al., 2009; Fliege, Lee, Grimm y Klapp, 2009; Hawton, Rodham, Evans y Weatherall, 2002; Lowenstein, 2005; Portzky y van Heeringen, 2007; Skegg, 2005; Stewart, Baiden y Theall-Honey, 2014; Swahn et al., 2012; Vajani, Annest, Crosby, Alexander y Millet, 2007; Valencia-Agudo, Burcher, Ezpeleta y Kramer, 2018). Debe reseñarse que el efecto de los síntomas depresivos es complejo, y que depende, no solo directamente de su presencia, si no de su intensidad, de la coexistencia con otros síntomas psicopatológicos asociados y del sexo de la persona que los sufre (Lundh, Wångby-Lundh y Bjärehed, 2011).

En cuanto al consumo de alcohol y drogas, salvo en algún estudio que no encuentra relación entre las conductas autolesivas y estas variables (Madge et al., 2008), su influencia directa de en las conductas autolesivas ha sido establecida de manera robusta (Balázs, Gyóri, Horváth, Mészáros y Szentiványi, 2018; Fulwiler, Forbes, Santangelo y Folstein, 1997; Heerde et al., 2015; Ilomäki, Räsänen, Viilo, Hakko y STUDY-70 Workgroup, 2007; Vargas-Martínez et al., 2018).

Respecto al resto de factores asociados a las DSH, los datos de la literatura son menos claros. El comportamiento prosocial es una conducta compleja resultado de la interacción entre aspectos intrapsíquicos y ambientales. Probablemente la búsqueda de asociación entre rasgos de personalidad y conducta suicida, que no se ha hecho en el presente estudio, aportase datos más concluyentes de asociación con las autolesiones, que el estudio de manifestaciones comportamentales como la conducta prosocial (Villar et al., 2018) u otras variables comportamentales como la falta de relaciones con compañeros, variable esta para la que la literatura también aporta datos discrepantes (Kaminski et al., 2010; Ruchkin, Sukhodolsky, Vermeiren, Koposov y Schwab-Stone, 2006).

Una situación parecida se da con la hiperactividad, que solo apareció asociado significativamente de forma débil a la conducta suicida en el estudio SEYLE. Estudios previos encontraron relación entre la hiperactividad como 
trastorno y las conductas autolesivas (Balázs et al., 2018; Bentley, Cassiello-Robbins, Vittorio, Sauer-Zavala y Barlow, 2015; Meszaros et al., 2017). También se ha postulado que la relación de la hiperactividad con las autolesiones pudiera ser indirecta a través de factores comunes como la impulsividad, como demostrarían los resultados de distintos estudios que, además, ponen de manifiesto que la asociación sería diferente en función del sexo (Hinshaw et al., 2012; Huang et al., 2017; Kashden, Fremouw, Callahan y Franzen, 1993; Meza, Owens y Hinshaw, 2016). Las características del presente estudio no permiten delimitar la naturaleza de la influencia de la hiperactividad o la impulsividad sobre las conductas autolesivas.

Finalmente, los consumos de drogas y tabaco aparecieron como factores significativamente asociados a las conductas autolesivas en el estudio SEYLE, pero no en WE-STAY. El caso del consumo de tabaco, merece consideración aparte, ya que durante el periodo de tiempo transcurrido entre los dos estudios hubo cambios sociales relacionados con el tabaquismo: un aumento del precio, la puesta en marcha de programas de prevención y sensibilización dirigidos a la población joven; y la promulgación de leyes restrictivas respecto al consumo (Ley 42/2010 de 30 de diciembre de 2010). Otros trabajos previos ya encontraron asociación entre consumo de tabaco y conductas autolesivas (Huang et al., 2017; Madge et al., 2011; Romero, Rodríguez, Villar y Gómez-Fraguela, 2017). La hipótesis de que las medidas tomadas en nuestro país encaminadas a la restricción del consumo de tabaco pudieran haber modificado la prevalencia de DSH en jóvenes es atractiva, pero para su confirmación precisaría del análisis de series temporales más largas.

El presente estudio presenta una serie de limitaciones entre las que se encuentran: que se trata de un análisis de datos retrospectivos y recogidos de modo autoinformado, que las poblaciones, siendo similares, no son totalmente equiparables, y que las poblaciones no son representativas de los adolescentes españoles, lo que no permite dar datos de prevalencia poblacional. Además, las diferencias en los datos entre dos estudios con metodología y población similares podrían suscitar dudas sobre la fiabilidad de los resultados. No obstante, cabe reseñar como principales fortalezas del presente trabajo, el hecho de que se analizan los resultados dos estudios metodológicamente similares, con muestras amplias y homogéneas y realizados con la solidez metodológica que garantizan los proyectos internacionales de los que deriva.

En conclusión, el análisis de dos estudios sobre conductas autolesivas en adolescentes, realizados con una metodología similar y en poblaciones de características sociodemográficas equiparables en espacios de tiempo próximos, muestra: que las tasas de DSH varían en función del estudio y del sexo en un rango entre $0,58 \%$ y $2,08 \%$; que los varones se autolesionan más frecuentemente mediante golpes autoinfligidos y quemaduras, mientras que las mujeres se hacen más frecuentemente cortes y escoriaciones; y que la presencia de síntomas depresivos y el consumo de alcohol son los factores asociados de forma más robusta a un mayor riesgo de DSH; ambos serían los factores prioritarios sobre los que incidir con campañas preventivas y de intervención.

\section{Reconocimientos}

Los proyectos SEYLE y WE-STAY han sido financiados por la Unión Europea a través del Séptimo Programa Marco (FP7), mediante los acuerdos de subvención número HEALTH-F2.2009-223091 y HEALTH-2010e241542, respectivamente. Los autores desean agradecer a todos los investigadores y personal que colaboró en ambos proyectos.

\section{Conflicto de intereses}

Los autores declaran que no existe conflicto de interés.

\section{Bibliografía}

Achenbach, T. M. (1991). Manual for the Youth Self-Report and 1991 Profile. Burlington, VT: University of Vermont Department of Psychiatry.

American Psychiatric Association. (2013). Diagnostic and Statistical Manual of Mental Disorders (DSM-5). Washington, DC: American Psychiatric Publishing.

Balázs, J., Győri, D., Horváth, L. O., Mészáros, G. y Szentiványi, D. (2018). Attention-deficit hyperactivity disorder and nonsuicidal self-injury in a clinical sample of adolescents: the role of comorbidities and gender. $B M C$ Psychiatry, 18, 34. doi:10.1186/s12888-018-1620-3.

Barrigón, M. L. y Baca-García, E. (2018). Retos actuales en la investigación en suicidio. Revista de Psiquiatría y Salud Mental, 11, 1-3. doi:10.1016/j.rpsm.2017.10.001.

Beck, A. T., Steer, R. A., Ball, R. y Ranieri, W. (1996). Comparison of Beck Depression Inventories -IA and -II in psychiatric outpatients. Journal of Personality Assessment, 67, 588-597. doi:10.1207/s15327752jpa6703_13.

Bentley, K. H., Cassiello-Robbins, C. F., Vittorio, L., Sauer-Zavala, S. y Barlow, D. H. (2015). The association between nonsuicidal self-injury and the emotional disorders: A meta-analytic review. Clinical Psychology Review, 37, 72-88. doi:10.1016/j.cpr.2015.02.006.

Bifulco, A., Schimmenti, A., Moran, P., Jacobs, C., Bunn, A. y Rusu, A. C. (2014). Problem parental care and teenage deliberate self-harm in young community adults. Bulletin of the Menninger Clinic, 78, 95-114. doi:10.1521/ bumc.2014.78.2.95.

Blasco-Fontecilla, H. (2018). Postmodernity, addictive societies, cannabis and suicidal behaviour: Towards a brave new world? Adicciones, 30, 3-8. doi:10.20882/adicciones. 1104 . 
Bousoño, M., Al-Halabí, S., Burón, P., Garrido, M., Díaz-Mesa, E. M., Galván, G., ... Bobes, J. (2017). Substance use or abuse, internet use, psychopathology and suicidal ideation in adolescents. Adicciones, 29, 97-104. doi:10.20882/adicciones.811.

Bousoño, M., Al-Halabí, S., Burón, P., Garrido, M., Díaz-Mesa, E. M., Galván, G., ... Bobes, J. (2019). Predictive factors of alcohol consumption in adolescents: data from 1-year follow-up prospective study. Adicciones, 31, 52-63. doi:10.20882/adicciones.998.

Bresin, K. y Schoenleber, M. (2015). Gender differences in the prevalence of nonsuicidal self-injury: A meta-analysis. Clinical Psychology Review, 38, 55-64. doi:10.1016/j. cpr.2015.02.009.

Brunner, R., Kaess, M., Parzer, P., Fischer, G., Carli, V., Hoven, C. W., ... Wasserman, D. (2014). Life-time prevalence and psychosocial correlates of adolescent direct self-injurious behavior: a comparative study of findings in 11 European countries. Journal of Child Psychology and Psychiatry, and Allied Disciplines, 55, 337-348. doi:10.1111/ jcpp. 12166.

Byrne, B. M., Stewart, S. M. y Lee, P. W. (2004). Validating the beck depression inventory-II for Hong Kong community adolescents. International Journal of Testing, 4, 199-216. doi:10.1207/s15327574ijt0403_1.

Carli, V., Hoven, C.W., Wasserman, C., Chiesa, F., Guffanti, G., Sarchiapone, M., ... Wasserman D. (2014). A newly identified group of adolescents at "invisible" risk for psychopathology and suicidal behavior: findings from the SEYLE study. World Psychiatry, 13, 78-86. doi:10.1002/ wps.20088.

Cipriano, A., Cella, S. y Cotrufo, P. (2017). Nonsuicidal self-injury: A systematic review. Frontiers in Psychology, 8, 1946. doi:10.3389/fpsyg.2017.01946.

Díaz de Neira, M., García-Nieto, R., de León-Martinez, V., Pérez Fominaya, M., Baca-García, E. y Carballo, J. J. (2015). Prevalence and functions of self-injurious thoughts and behaviors in a sample of Spanish adolescents assessed in mental health outpatient departments. Revista de Psiquiatria y Salud Mental, 8, 137-145. doi:10.1016/j. rpsm.2013.09.003.

Dougherty, D. M., Mathias, C. W., Marsh-Richard, D. M., Prevette, K. N., Dawes, M. A., Hatzis, E. S., ... Nouvion, S. O. (2009). Impulsivity and clinical symptoms among adolescents with non-suicidal self-injury with or without attempted suicide. Psychiatry Research, 169, 22-27. doi:10.1016/j.psychres.2008.06.011.

Fliege, H., Lee, J.R., Grimm, A. y Klapp, B. F. (2009). Risk factors and correlates of deliberate self-harm behavior: a systematic review. Journal of Psychosomatic Research, 66, 477-493. doi:10.1016/j.jpsychores.2008.10.013.

Fonseca-Pedrero, E., Inchausti, F., Pérez-Gutiérrez, L., Aritio Solana, R., Ortuño-Sierra, J., Sánchez-García, M. Á., ... Pérez de Albéniz Iturriaga, A. (2018). Ideación suicida en una muestra representativa de adolescentes españoles. Revista de Psiquiatría y Salud Mental, 11, 76-85. doi:10.1016/j.rpsm.2017.07.004.

Fulwiler, C., Forbes, C., Santangelo, S. L. y Folstein, M. (1997). Self-mutilation and suicide attempt: distinguishing features in prisoners. The Journal of the American Academy of Psychiatry and the Law, 25, 69-77.

Goodman, R., Meltzer, H. y Bailey, V. (2003). The Strengths and Difficulties Questionnaire: a pilot study on the validity of the self-report version. International Review of Psychiatry, 15, 173-177. doi:10.1080/0954026021000046137.

Gratz, K. L. (2001). Measurement of Deliberate Self-Harm: Preliminary Data on the Deliberate Self-Harm Inventory. Journal of Psychopathology and Behavioral Assessment, 23, 253-263. doi:10.1023/A:1012779403943.

Hargus, E., Hawton, K. y Rodham, K. (2009). Distinguishing between subgroups of adolescents who selfharm. Suicide E Life-Threatening Behavior, 39, 518-537. doi:10.1521/suli.2009.39.5.518.

Hawton, K. y Harriss, L. (2008). Deliberate self-harm by under-15-year-olds: characteristics, trends and outcome. Journal of Child Psychology and Psychiatry, and Allied Disciplines, 49, 441-448. doi:10.1111/j.1469-7610.2007.01852.x.

Hawton, K., Rodham, K., Evans, E. y Weatherall, R. (2002). Deliberate self-harm in adolescents: self-report survey in schools in England. British Medical Journal, 325, $1207-$ 1211.

Heerde, J. A., Toumbourou, J. W., Hemphill, S. A., Herrenkohl, T. I., Patton, G. C. y Catalano, R. F. (2015). Incidence and Course of Adolescent Deliberate Self-Harm in Victoria, Australia, and Washington State. Journal of Adolescent Health, 57, 537-544. doi:10.1016/j.jadohealth.2015.07.017.

Hinshaw, S. P., Owens, E. B., Zalecki, C., Huggins, S. P., Montenegro-Nevado, A. J., Schrodek, E. y Swanson, E. N. (2012). Prospective follow-up of girls with attention-deficit/hyperactivity disorder into early adulthood: continuing impairment includes elevated risk for suicide attempts and self-injury. Journal of Consulting and Clinical Psychology, 80, 1041-1051. doi:10.1037/a0029451.

Huang, Y.H., Liu, H.-C., Tsai, F.-J., Sun, F.-J., Huang, K.-Y., Chiu, Y.-C., ... Liu, S.-I. (2017). Correlation of impulsivity with self-harm and suicidal attempt: a community study of adolescents in Taiwan. BMJ Open, 7, e017949. doi:10.1136/bmjopen-2017-017949.

Ilomäki, E., Räsänen, P., Viilo, K., Hakko, H. y STUDY-70 Workgroup. (2007). Suicidal behavior among adolescents with conduct disorder-the role of alcohol dependence. Psychiatry Research, 150, 305-311. doi:10.1016/j. psychres.2006.02.011.

Kaess, M., Fischer-Waldschmidt, G., Resch, F. y Koenig, J. (2017). Health related quality of life and psychopathological distress in risk taking and self-harming adolescents with full-syndrome, subthreshold and without 
M. Bousoño, S. Al-Halabí, P. Burón, M. Garrido, E. Mª Díaz-Mesa, G. Galván, L. García-Álvarez, Á. Velasco, J. Rodríguez-Revuelta, C. Wasserman, V. Carli, C. Hoven, M. Sarchiapone, D. Wasserman, M. Bousoño, Mª P. García-Portilla, C. Iglesias, P. Alejandra Sáiz, J. Bobes

borderline personality disorder: rethinking the clinical cut-off? Borderline Personality Disorder and Emotion Dysregulation, 4, 7. doi:10.1186/s40479-017-0058-4.

Kaminski, J. W., Puddy, R. W., Hall, D. M., Cashman, S. Y., Crosby, A. E. y Ortega, L. A. G. (2010). The relative influence of different domains of social connectedness on self-directed violence in adolescence. Journal of Youth and Adolescence, 39, 460-473. doi:10.1007/s10964-009-9472-2.

Kashden, J., Fremouw, W. J., Callahan, T. S. y Franzen, M. D. (1993). Impulsivity in suicidal and nonsuicidal adolescents. Journal of Abnormal Child Psychology, 21, 339-353.

Kirchner, T., Ferrer, L., Forns, M. y Zanini, D. (2011). Selfharm behavior and suicidal ideation among high school students. Gender differences and relationship with coping strategies. Actas Españolas de Psiquiatría, 39, 226-235.

Lenkiewicz, K., Racicka, E. y Bryńska, A. (2017). Self-injury - placement in mental disorders classifications, risk factors and primary mechanisms. Review of the literature. Psychiatria Polska, 51, 323-334. doi:10.12740/PP/62655.

Lowenstein, L. F. (2005). Youths who intentionally practise self-harm. Review of the recent research 2001-2004. International Journal of Adolescent Medicine and Health, 17, 225-230

Lundh, L.G., Wångby-Lundh, M. y Bjärehed, J. (2011). Deliberate self-harm and psychological problems in young adolescents: evidence of a bidirectional relationship in girls. Scandinavian Journal of Psychology, 52, 476-483. doi:10.1111/j.1467-9450.2011.00894.x.

Madge, N., Hawton, K., McMahon, E. M., Corcoran, P., De Leo, D., de Wilde, E. J., ... Arensman, E. (2011). Psychological characteristics, stressful life events and deliberate self-harm: findings from the Child \& Adolescent Self-harm in Europe (CASE) Study. European Child E Adolescent Psychiatry, 20, 499-508. doi:10.1007/s00787011-0210-4.

Madge, N., Hewitt, A., Hawton, K., de Wilde, E. J., Corcoran, P., Fekete, S., ... Ystgaard, M. (2008). Deliberate self-harm within an international community sample of young people: comparative findings from the Child \& Adolescent Self-harm in Europe (CASE) Study. Journal of Child Psychology and Psychiatry, and Allied Disciplines, 49, 667-677. doi:.10.1111/j.1469-7610.2008.01879.x.

McCormick, E. M., Qu, Y. y Telzer, E. H. (2017). Activation in context: Differential conclusions drawn from cross-sectional and longitudinal analyses of adolescents' cognitive control-related neural activity. Frontiers in Human Neuroscience, 11, 141. doi:10.3389/fnhum.2017.00141.

Meszaros, G., Horvath, L. O. y Balazs, J. (2017). Self-injury and externalizing pathology: a systematic literature review. BMC Psychiatry, 17, 160. doi:10.1186/s12888-0171326-y.

Meza, J. I., Owens, E. B. y Hinshaw, S. P. (2016). Response inhibition, peer preference and victimization, and selfharm: Longitudinal associations in young adult women with and without ADHD. Journal of Abnormal Child Psychology, 44, 323-334. doi:10.1007/s10802-015-0036-5.

Morgan, C., Webb, R. T., Carr, M. J., Kontopantelis, E., Green, J., Chew-Graham, C. A., ... Ashcroft, D. M. (2017). Incidence, clinical management, and mortality risk following self-harm among children and adolescents: cohort study in primary care. British Medical Journal, 359, j4351. doi:10.1136/bmj.j4351.

Muehlenkamp, J. J., Claes, L., Havertape, L. y Plener, P. L. (2012). International prevalence of adolescent non-suicidal self-injury and deliberate self-harm. Child and Adolescent Psychiatry and Mental Health, 6, 10. doi:10.1186/1753-2000-6-10.

Nock, M. K. (2010). Self-injury. Annual Review of Clinical Psychology, 6, 339-363. doi:10.1146/annurev. clinpsy.121208.131258.

Pattison, E. M. y Kahan, J. (1983). The deliberate self-harm syndrome. The American Journal of Psychiatry, 140, 867872. doi:10.1176/ajp.140.7.867.

Pisinger, V. S. C., Hawton, K. y Tolstrup, J. S. (2018). Self-injury and suicide behavior among young people with perceived parental alcohol problems in Denmark: a school-based survey. European Child E Adolescent Psychiatry, 27, 201-208. doi:10.1007/s00787-017-1031-x.

Portzky, G. y van Heeringen, K. (2007). Deliberate selfharm in adolescents. Current Opinion in Psychiatry, 20, 337-342. doi:10.1097/YCO.0b013e3281c49ff1.

Romero, E., Rodríguez, C., Villar, P. y Gómez-Fraguela, X. A. (2017). Intervention on early-onset conduct problems as indicated prevention for substance use: A seven-year follow up. Adicciones, 29, 150-162. doi:10.20882/adicciones.722.

Ruchkin, V., Sukhodolsky, D. G., Vermeiren, R., Koposov, R. A. y Schwab-Stone, M. (2006). Depressive symptoms and associated psychopathology in urban adolescents: A cross-cultural study of three countries. Journal of Nervous and Mental Disease, 194, 106-113. doi:10.1097/01. nmd.0000198142.26754.18.

Silverman, M. M., Berman, A. L., Sanddal, N. D., O’Carroll, P. W. y Joiner, T. E. (2007). Rebuilding the tower of Babel: a revised nomenclature for the study of suicide and suicidal behaviors. Part 2: Suicide-related ideations, communications, and behaviors. Suicide E Life-Threatening Behavior, 37, 264-277. doi:10.1521/suli.2007.37.3.264.

Skegg, K. (2005). Self-harm. Lancet, 366, 1471-1483. doi:10.1016/S0140-6736(05)67600-3.

Somerville, L. H., Hare, T. y Casey, B. J. (2011). Frontostriatal maturation predicts cognitive control failure to appetitive cues in adolescents. Journal of Cognitive Neuroscience, 23, 2123-2134. doi:10.1162/jocn.2010.21572.

Sornberger, M. J., Heath, N. L., Toste, J. R. y McLouth, R. (2012). Nonsuicidal self-injury and gender: patterns of prevalence, methods, and locations among adoles- 
cents. Suicide Eं Life-Threatening Behavior, 42, 266-278. doi:10.1111/j.1943-278X.2012.0088.x.

Stewart, S. L., Baiden, P. y Theall-Honey, L. (2014). Examining non-suicidal self-injury among adolescents with mental health needs, in Ontario, Canada. Archives of Suicide Research, 18, 392-409. doi:10.1080/13811118.2013.8 24838.

Strittmatter, E., Kaess, M., Parzer, P., Fischer, G., Carli, V., Hoven, C.W., ... Wasserman D. (2015). Pathological Internet use among adolescents: Comparing gamers and non-gamers. Psychiatry Research, 228, 128-135. doi:10.1016/j.psychres.2015.04.029.

Swahn, M. H., Ali, B., Bossarte, R. M., Van Dulmen, M., Crosby, A., Jones, A. C. y Schinka, K. C. (2012). Selfharm and suicide attempts among high-risk, urban youth in the U.S.: shared and unique risk and protective factors. International Journal of Environmental Research and Public Health, 9, 178-191. doi:10.3390/ijerph9010178.

Taliaferro, L. A. y Muehlenkamp, J. J. (2015). Risk factors associated with self-injurious behavior among a national sample of undergraduate college students. Journal of American College Health, 63, 40-48. doi:10.1080/07448 481.2014 .953166$.

Vajani, M., Annest, J. L., Crosby, A. E., Alexander, J. D. y Millet, L. M. (2007). Nonfatal and fatal self-harm injuries among children aged 10-14 years-United States and Oregon, 2001-2003. Suicide E Life-Threatening Behavior, 37, 493-506. doi:10.1521/suli.2007.37.5.493.

Valencia-Agudo, F., Burcher, G. C., Ezpeleta, L. y Kramer, T. (2018). Nonsuicidal self-injury in community adolescents: A systematic review of prospective predictors, mediators and moderators. Journal of Adolescence, 65, 25-38. doi:10.1016/j.adolescence.2018.02.012.
Vargas-Martínez, A. M., Trapero-Bertran, M., Gil-García, E. y Lima-Serrano, M. (2018). Impact of the Binge Drinking (BD) in Adolescence. Are we doing it right? Adicciones, 30, 152-154. doi:10.20882/adicciones.1033.

Villar, F., Letemendia, E. E., Blasco, T., Toribio, T. P., Verge, M., Grifoll, M. del M. V., ... Tejedor, C. C. (2018). Análisis dimensional de la personalidad del adolescente con conducta suicida. Actas Españolas de Psiquiatría, 46, 104-111.

Wasserman, D., Carli, V., Wasserman, C., Apter, A., Balazs, J., Bobes, J., ... Hoven, C. W. (2010). Saving and Empowering Young Lives in Europe (SEYLE): A randomized controlled trial. BMC Public Health, 10, 192. doi:10.1186/1471-2458-10-192.

Whitlock, J., Muehlenkamp, J., Purington, A., Eckenrode, J., Barreira, P., Baral Abrams, G., ... Knox, K. (2011). Nonsuicidal self-injury in a college population: general trends and sex differences. Journal of American College Health, 59, 691-698. doi:10.1080/07448481.2010.529626.

World Health Organization. (2015). Global School-Based Student Health Survey. Recuperado de http://www. who.int/chp/gshs/GSHS_Questionnaire_Core_2009_ Spanish.pdf.

Yang, X. y Feldman, M. W. (2017). A reversed gender pattern? A meta-analysis of gender differences in the prevalence of non-suicidal self-injurious behaviour among Chinese adolescents. BMC Public Health, 18, 66. doi:10.1186/s12889-017-4614-z.

Zetterqvist, M. (2015). The DSM-5 diagnosis of nonsuicidal self-injury disorder: a review of the empirical literature. Child and Adolescent Psychiatry and Mental Health, 9, 31. doi:10.1186/s13034-015-0062-7. 